\title{
Cerebral Small Vessel Disease Clinical, Neuropsychological, and Radiological Phenotypes, Histopathological Correlates, and Described Genotypes: A Review
}

\author{
Thomas Gregor Issac, ${ }^{1}$ Sadandavalli Retnaswami Chandra, ${ }^{2}$ Rita Christopher, ${ }^{3}$ \\ Jamuna Rajeswaran, ${ }^{4}$ and Mariamma Philip ${ }^{5}$ \\ ${ }^{1}$ Clinical Neurosciences, National Institute of Mental Health and Neurosciences, Bangalore 560029, India \\ ${ }^{2}$ Department of Neurology, National Institute of Mental Health and Neurosciences (NIMHANS), Bangalore 560029, India \\ ${ }^{3}$ Department of Neurochemistry, National Institute of Mental Health and Neurosciences (NIMHANS), Bangalore 560029, India \\ ${ }^{4}$ Department of Clinical Psychology, National Institute of Mental Health and Neurosciences (NIMHANS), Bangalore 560029, India \\ ${ }^{5}$ Department of Biostatistics, National Institute of Mental Health and Neurosciences (NIMHANS), Bangalore 560029, India
}

Correspondence should be addressed to Sadandavalli Retnaswami Chandra; drchandrasasi@yahoo.com

Received 12 August 2015; Revised 9 December 2015; Accepted 10 December 2015

Academic Editor: Stephen D. Ginsberg

Copyright (C) 2015 Thomas Gregor Issac et al. This is an open access article distributed under the Creative Commons Attribution License, which permits unrestricted use, distribution, and reproduction in any medium, provided the original work is properly cited.

\begin{abstract}
Introduction. Vascular cognitive impairment is a common yet preventable cause for dementia. It needs high degree of suspicion and appropriate designing of investigatory tools to confirm diagnosis, identify comorbidities, and ascertain the areas of impairment. Commonly DSM-IV criterion is applied for diagnosis and detailed clinical and neuropsychological examination for identifying the specific phenotype is used. Early diagnosis using the mandatory criteria will help in early initiation of disease modifying treatment strategies which can result in partial reversal of vascular changes and arrest of progression. Patients with young onset disease might require genetic characterization for designing more aggressive treatment. Discussion and Conclusion. Dementias as such carry poor course and prognosis resulting in severe Disability Adjusted Life Years (DALYs) for patients and caregivers. Therefore, it is mandatory to identify treatable and preventable causes so that man power loss can be reduced.
\end{abstract}

\section{Introduction}

Cerebral small vessel disease (SVD) is characterized by disease of the small perforating arteries which are defined as "vessels with a diameter less than $50 \mu \mathrm{m}$ " and "all the vessels within the brain parenchyma plus the vessels with a diameter less than $500 \mu \mathrm{m}$ in the leptomeningeal space" supplying the deep brain structures [1]. It is the most important and common cause of vascular dementia accounting for more than $40 \%$ of the total prevalence. The term vascular cognitive impairment was proposed by Sachdev [2] as an umbrella term to denote cognitive disorders due to cerebrovascular cause ranging from Mild Cognitive Impairment stage (MCI) to vascular dementia and cerebral SVD is an important and major contributor to the same $[1,3]$. Since cerebral SVD is difficult to detect in vivo, we have to rely on radiological markers for the same and predominantly the triad of Periventricular white matter hyperintensities, lacunes, and microbleeds constitute the continuum of SVD $[3,4]$. The newer neuropathological correlates of the lesions ascribed to SVD also have immensely helped in understanding the pathophysiology of this hitherto common but often missed disease $[1,5]$. Unlike other degenerative dementias of cortical and subcortical origin, vascular dementia is partially reversible and preventable.

Cerebral SVD is not a rare entity. It was even described by the ancient Indian Physician Charaka as Smritbhramsha due to the hardening of the Dhamani or the blood vessel ultimately resulting in the hampering of the flow of Rasa and Rakta to the brain tissue leading to cognitive impairment [6, 7]. Later, the work of Otto Binswanger and Alois Alzheimer 
TABLE 1: NINDS-AIREN and ADDTC criteria for vascular dementia.

\begin{tabular}{|c|c|c|}
\hline & ADDTC & NINDS-AIREN \\
\hline Dementia definition & $\begin{array}{l}\text { Deterioration from a known level of intellectual } \\
\text { function sufficient to interfere with the patients } \\
\text { customary affairs of life and which is not isolated to a } \\
\text { single category of intellectual performance. }\end{array}$ & $\begin{array}{l}\text { Impairment of memory plus at least two other areas of } \\
\text { cognitive domains, which should be severe enough to } \\
\text { interfere with activities of daily living and not due to } \\
\text { physical effects of stroke alone. }\end{array}$ \\
\hline Probable VaD & $\begin{array}{l}\text { Requiring all of the following: } \\
\text { Dementia. } \\
\text { Evidence of two or more strokes by history, neurological } \\
\text { signs, and/or imaging, or a single stroke with a clear } \\
\text { temporal relationship to the onset of dementia. } \\
\text { Evidence of at least one infarct outside the cerebellum } \\
\text { by CT or T1 weighted MRI. }\end{array}$ & $\begin{array}{l}\text { Requiring all of the following: } \\
\text { Dementia. } \\
\text { CVD: focal signs on examination + evidence of relevant } \\
\text { CVD by CT/MRI. } \\
\text { A relationship between the above two disorders, } \\
\text { manifested by one or more of the following: } \\
\text { Dementia onset within } 3 \text { months of a stroke. } \\
\text { Abrupt deterioration in cognitive function, or } \\
\text { fluctuating stepwise course. }\end{array}$ \\
\hline Possible VaD & $\begin{array}{l}\text { Dementia and one of the following: } \\
\text { History or evidence of a single stroke without a clear } \\
\text { temporal relationship to the dementia onset. } \\
\text { Binswanger's disease that includes all the following: } \\
\text { early onset of urinary incontinence or gait disturbance; } \\
\text { vascular risk factors; extensive white matter changes on } \\
\text { neuroimaging. }\end{array}$ & $\begin{array}{l}\text { May be made in the presence of dementia and focal } \\
\text { neurological signs in patients either with no evidence of } \\
\text { CVD on neuroimaging, with the absence of a clear } \\
\text { temporal relationship between stroke and dementia, or } \\
\text { with subtle onset and variable course of cognitive deficit } \\
\text { and evidence of CVD. }\end{array}$ \\
\hline
\end{tabular}

ADDTC: State of California Alzheimer's Disease Diagnostic and Treatment Centers; CT: computed tomography; CVD: cerebrovascular disease; MRI: magnetic resonance imaging; NINDS-AIREN: National Institute of Neurological Diseases and Stroke and Association Internationale pour la Recherché et l'Enseignement en Neurosciences; VaD: vascular dementia.

in late 1897 differentiated vascular causes of dementia from neurosyphilis [8, 9]. In 1910 Kreaplin concluded that insanity due to arteriosclerosis was the most frequent form of senile dementia [10]. By 1970s the focus had shifted over to Alzheimer's disease but Tomlinson, Blessed, and Roth showed that the loss of more than $50-100 \mathrm{~mm}^{3}$ of brain tissue consequent on strokes caused cognitive impairment and was referred to as multi-infarct dementia [11]. Diagnostic criteria commonly applied are DSM-IV and NINDS-AIREN criteria and Hachinski's ischemic score (Tables 1 and 2 and Box 1).

C. M. Fisher did the maximum work on the clinicopathological studies on cerebral SVD and is credited for coining the term lacune as well as describing the Fishers rule which implicates that for every single cerebral vessel which is affected two coronaries and three peripheral vessels are already affected. He was thus the pioneer in research in vascular dementia, especially with regard to cerebral SVD. Unlike large vessel disease, even though there is a progressive chronic course in SVD, a subacute onset is often described in SVD due to lacunar state. A chronic progressive course behaving as a progressive frontotemporal syndrome was previously referred to as Binswanger's disease.

Cerebral SVD is often classified on the basis of etiology into 5 major groups which include the following.

Type 1. Arteriolosclerosis (or age and vascular risk factorsrelated SVD).

Type 2. Cerebral amyloid angiopathy (sporadic and hereditary).
TABLE 2: Hachinski's ischemic score. Purpose: the Hachinski ischaemic score (HIS) represents a brief clinical tool helpful in the "bedside" differentiation of the commonest dementia types, Dementia of Alzheimer's Type (DAT) and Vascular Dementia (VaD). A cutoff score $\leq 4$ for DAT and $\geq 7$ for VaD has a sensitivity of $89 \%$ and a specificity of $89 \%$ [23].

\begin{tabular}{llc}
\hline Item number & Description & Value \\
\hline 1 & Abrupt onset & 2 \\
2 & Stepwise deterioration & 1 \\
3 & Fluctuating course & 2 \\
4 & Nocturnal confusion & 1 \\
5 & Preservation of personality & 1 \\
6 & Depression & 1 \\
7 & Somatic complaints & 1 \\
8 & Emotional incontinence & 1 \\
9 & History of hypertension & 1 \\
10 & History of stroke & 2 \\
11 & Associated atherosclerosis & 1 \\
12 & Focal neurological symptoms & 2 \\
13 & Focal neurological signs & 2 \\
\hline
\end{tabular}

Reference: Moroney et al. [23].

Type 3. Inherited or genetic SVD (distinct from cerebral amyloid angiopathy, e.g., CADASIL, CARASIL, Fabry's disease, and SVD due to COL4A1 mutations).

Type 4. Inflammatory and immunomediated SVD (systemic and cerebral vasculitis, central nervous system vasculitis secondary to infections). 
(A) The development of multiple cognitive deficits manifested by both

(1) Memory impairment (impaired ability to learn new information or to recall previously learned information)

(2) One or more of the following cognitive disturbances:

(a) Aphasia (language disturbance)

(b) Apraxia (impaired ability to carry out motor activities despite intact motor function)

(c) Agnosia (failure to recognize or identify objects despite intact sensory function)

(d) Disturbance in executive functioning (i.e., planning, organizing, sequencing, and abstracting)

(B) The cognitive deficits in criteria (A) - (1) and (2) each cause significant impairment in social or occupational functioning and represent a significant decline from a previous level of functioning.

(C) Focal neurological signs and symptoms (e.g., exaggeration of deep tendon reflexes, extensor plantar response, pseudobulbar palsy, gait abnormalities, and weakness of an extremity) or laboratory evidence indicative of cerebrovascular disease (e.g., multiple infarctions involving cortex and underlying white matter) that are judged to be etiologically related to the disturbance.

(D) The deficits do not occur exclusively during the course of a delirium.

Box 1: DSM-IV criteria for vascular dementia.

Type 5. Venous collagenosis.

Type 6. Other SVD (e.g., postradiation angiopathy and nonamyloid microvessel degeneration in Alzheimer's disease).

Currently cerebral SVD is a neuroimaging defined concept and there has been a unified approach to the neuroimaging standards for research in SVD under the acronym STRIVE (STandards for ReportIng Vascular changes on nEuroimaging) which has resulted in the adaptation of major four conclusions which include the following: (A) the number of SVD lesion subtypes was broadened to include six types of neuroimaging lesions: (1) recent small subcortical infarcts; (2) lacunes of presumed vascular origin; (3) white matter hyperintensity of presumed vascular origin; (4) perivascular spaces; (5) cerebral microbleeds; and (6) brain atrophy based on the association between subcortical lesions and brain atrophy; (B) the establishment of a common language about terms and definitions for SVD features visible on magnetic resonance imaging (MRI); (C) maintenance of minimum standards for image acquisition and analysis; (D) agreement on standards for scientific reporting of changes related to SVD on neuroimaging along with review of the newer imaging techniques for the detection and quantification of preclinical manifestations of SVD $[1,3]$.

Presence of long term hypertension was identified as the most important risk factor with regards to the same and the role of renin-angiotensin-aldosterone both internal and external to the blood brain barrier is postulated to maintain an important homeostatic role determining the progression of SVD [1, 3, 12-15].

This review article focusses mainly on the clinical manifestations of sporadic SVD referring to a heterogeneous group of phenotypes based on brain imaging, their neuropsychological correlates, the common radiological findings seen in both CT scan and MR imaging, and the scales used to quantify the same and some described genetic mechanisms especially both important monogenic causes of SVD as well as the role of certain specific pathways like the renin-angiotensinaldosterone system in the genesis and evolution of the disease condition. The word sporadic SVD is mentioned but in view of the gross variation in clinical symptomatology in the presence of similar grade of radiological features, we believe that there is a probably genetic factor hitherto unidentified that could be causally involved. This review article deals with pure vascular dementias and therefore other dementias associated with vascular changes and mixed dementias have not been considered.

\section{Clinical Manifestations}

Patients presenting with SVD usually present in their later decades with often features suggestive of cognitive slowing, psychomotor withdrawal due to severe executive dysfunction described as difficulty to pay attention, retrieval defect, tip of the tongue phenomena, short term memory defects, and occasionally impaired judgement [16]. Gait and balance disturbances and falls are also major symptoms of patients with cerebral small vessel disease (SVD), and an important cause of morbidity and mortality in the elderly. Motor deficits associated with SVD present in different ways, such as unsteadiness of gait, impaired balance on walking, and increased risk of falls. Furthermore, gait abnormalities have been shown to predict non-Alzheimer's dementia, that is, mainly vascular dementia. A $22 \%$ greater risk for falls for those with moderate white matter lesions (WMLs) was seen than for those with minimal WMLs, and a $26 \%$ greater risk for participants with at least one brain infarct than for those with no infarcts. Interestingly, participants with moderate to severe brain abnormalities experienced faster gait speed decline and features suggestive of lower segment parkinsonism. Soumaré et al. postulated a threshold effect of WMH load: a pathologic change in the white matter will be compensated up to a certain degree without leading to functional decline. WMH load stands for visual quantification of the white matter changes based on well-defined criteria. Once this threshold is surpassed, subjects will show impairment. Periventricular and deep frontal WMHs were found to be independently associated with falls $[1,16]$. The key link between WMLs and fall risk therefore is the impairment of balance control: compared with subjects without a history 
of fall, the mean single leg stance time was significantly reduced in individuals with a history of a single or multiple falls. WMH also acts as an independent predictor of physical decline and cognitive impairment. Vascular dementia is seen more commonly in males than in females in view of their more proneness for comorbidities. Elderly females are more affected by Alzheimer's disease than males and relatively younger males are more affected by Frontotemporal dementia than females [17].

The psychiatric symptoms include apathy and depression. The relationship between SVD and depression does not necessarily have to be one-sided. While SVD could arguably lead to depression, the reverse may also be true as depression has been seen as a risk factor for vascular disease. Radiological evidence for white matter pathology is seen in wide variety of disorders as well as in normal people. However, our article review consists of only those with phenotypically and radiologically confirmed small vessel disease patients only and other groups of disorders are not being considered in this review. Obsessive Compulsive Disorder (OCD) is the subject of a large number of neuroimaging and neuropsychological studies. WMHs have been described in early-onset OCD. Late-onset OCD is, however, uncommon, with only $15 \%$ presenting after the age of 35 , and neuroimaging data on these are not available. Late-onset OCD has been associated with more frequent brain lesions, including silent strokes, with lesions largely being in the cerebral cortex or the basal ganglia. Neuroimaging findings in OCD have suggested an orbital-frontal-striatal circuit underlying the disorder, and microstructural abnormalities of white matter demonstrated on DTI have added weight to the underlying disruption of a circuit comprising of frontal, striatal, and thalamic connectivity.

Apathy is conceptualized as a diminution of motivation but is variously described as a symptom as well as a syndrome, combining emotional, behavioral, and cognitive components. Levy and Dubois emphasized the observable behavioral components arising from disruptions in the basal ganglia and prefrontal cortex. Apathy is often mistaken for depression, although the correlation between apathy and depression ratings is often low, and the neuropsychological profiles differ $[1,13,16]$.

They usually have associated comorbidities like hypertension, diabetes mellitus, hypercholesterolemia, history of ischemic cardiac illness, and renal diseases along with history of substance abuse especially alcohol and tobacco. Hypertension is the most important risk factor for development of cerebral SVD. The neurological manifestations of SVD consist of a wide spectrum of clinical phenotypes which commonly includes the lacunar strokes like (1) pure motor hemiparesis, (2) pure sensory hemiparesis, (3) ataxic hemiparesis, (4) dysarthria clumsy hand syndrome, and (5) pseudobulbar syndrome. Apart from these there are features of executive dysfunction, short term memory loss, mood changes, and autonomic dysfunction which has been described $[1,5,6,18]$.

A small note has to be maintained regarding the prodromal phase of subclinical to clinical depression prior to the onset of the illness when the patient is detached, uninterested, and often having poor attention span which is often seen. These features can be together described as "vascular depression." Whether this depression is a cause or a consequence of cerebral SVD is still not fully known although many studies have implicated a positive correlation $[5,6,19]$.

\section{Neuropsychological Correlates of SVD}

As described before, executive dysfunction with retrieval defect is the most common neuropsychological finding seen in patients with cerebral SVD. Executive function refers to those higher cognitive processes by which performance is optimized in situations requiring the simultaneous operation of several cognitive processes. Usually the patients with SVD have difficulty in multitasking, a common feature associated with normal ageing along with apathy, disinterest, poor attention, and often irritability. Therefore, executive skills which are used to construct effective plans of action, particularly in the face of complex tasks, are affected most in SVD and have to be distinguished from the benign forgetfulness and executive dysfunction associated with normal ageing. Executive dysfunction, mainly characterized by deficits in set shifting, verbal fluency, abstract problem solving, attention, and decreased speed of information processing, might be detectable even in the initial stages of the disease. Multiple lacunes located in the subcortical structures and confluent and extensive WMCs can result in a disconnection of frontalsubcortical circuits (in particular, the dorsolateral prefrontal circuit) interrupting the central cholinergic pathway causing impairment of the working memory and Judgement. Patients may need repeated instructions to complete tasks and are easily distractible. Due to poor attention and impaired working memory new verbal and visual learning is usually affected $[6,18,20]$. Mental and motor slowing can also be seen in patients with cerebral SVD [19]. The MMSE test is of not much value in assessment of SVD as per previous studies as the items in MMSE are grossly insensitive to the executive functions [20]. Hence the following neuropsychological tests are mandatory to assess SVD. This includes digit forward and backward test, trail making tests to assess attention and working memory. The category fluency test is used for fund of knowledge or generativity, the digit symbol substitution test to assess focused attention and performance IQ, the stick test to assess visual recall and working memory, the passage or story telling test for verbal recall and learning, and the Bender Gestalt test to determine organicity. Digit forward and backward tests involve asking the patient to repeat random numbers told by the examiner in the same order as well as from the last to the first. Digit forward test involves mostly attention and digit backward test involves working memory. Trail making test involves type A where patient has to connect numbers in that order without crisscrossing. Errors of commission and time taken are calculated. Trail making 2 test involves little more complex testing where each number has to be connected to a color in an alternating fashion. Category fluency test involves asking the patient to tell the maximum number of words he can generate starting with the letters F, A, and S in one minute. Digit symbol substitution test involves patient rapidly putting the designated numbers given in the test kit below each test symbol. 
Errors and timing are calculated. Stick test involves showing the nine standardized pictures formed using 5 straight lines, each shown for 30 seconds. After that patient is asked to reproduce those figures from memory using 5 matchsticks. This test evaluates registration and delayed recall. Passage test involves telling the patient a well validated story designated as "Asha Kumari story" which contains 21 parameters to be registered and recalled by the patient. Bender Gestalt test involves nine cards containing pictures of increasing complexity which the patient has to copy and is normally not affected in patients with nonorganic problems [21]. Executive and activation functions are frequently assessed using timed tests with a variety of set shifting, mental flexibility, and response inhibition tasks. Both executive functions and speed of processing are frequently impaired in patients with SVD. Lower scores on verbal fluency, Stroop reading and color naming tasks, and trail making tests have been repeatedly associated with SVD. On other nontimed instruments used to assess mental flexibility and conceptualization, such as the Wisconsin Card Sorting Test, changes on scores were also associated with progression of white matter changes (WMC) in healthy community-dwelling elderly volunteers. On a brief assessment battery used to differentiate patients with ischemic leukoaraiosis from normal controls, trail making and digit symbol substitution tasks were the best individual tests identifying cognitive impairment. Also on other studies, a decline in the digit symbol substitution test performance was associated with worsening and progression of WMCs. Tests sensitive to reductions in psychomotor speed, mental flexibility, working memory, and attention, such as the digit symbol or trail making, are useful instruments for detecting early subtle changes in SVD patients.

Memory deficits observed in SVD are distinctive from those observed in other pathologies such as MCI and AD. Studies comparing patients with MCI, with and without SVD, tried to identify different cognitive profiles. Zhou et al. reported a nonspecific profile in MCI-SVD patients, since they found extensive cognitive deficits in these patients. Comparatively, patients with MCI-AD had a more specific pattern, characterized by a significantly worse performance on verbal and visual delay recall and recognition measures, with a relatively preserved performance on processing speed tasks. MCI patients with severe WMCs were compared with MCI patients with severe hippocampal atrophy using a comprehensive neuropsychological battery. The authors found that patients with hippocampal atrophy exhibited specific episodic memory impairment, while patients with WMCs exhibited deficits on episodic memory (assessed by a 36-item object color-association task), working memory (patients were tested on two verbal working memory tasks and a spatial working memory task), and attention-control tasks (continuous performance test), suggesting that the episodic memory deficit was secondary to a more general impairment of executive control. In fact, working memory performance requires an ability to hold, manipulate, and quickly access information that is dependent on mental flexibility and speed. The mediation effect of executive functions on verbal and visual memory performance of SVD patients is already described. Distinctive patterns can be observed in relation to memory impairment. Ability to store information is mainly dependent on the limbic and hippocampal structures, while retrieval and short term memory capacities are more related with the integrity of frontal-subcortical structures and temporoparietal regions, respectively. In SVD patients, episodic memory impairment is usually absent or mild in the initial phases, but in later stages is described as being the most frequent deficit characterized by difficulty in recalling information that improves in the presence of cues. However, these findings are frequently associated with other variables, such as medial temporal lobe atrophy and corpus callosum atrophy. Poorer episodic memory has been associated with age related differences in central white matter regions. Recent studies with SVD patients have explored the relationship of microstructural integrity of the hippocampus, not detected with conventional MRI, and verbal memory impairment. These authors found that deficits in verbal memory (immediate, delayed recall, and delayed recognition), but not in psychomotor speed, were related to changes in the hippocampus microstructure and, consequently, in the cingulum. These results might lead us into a new perspective in the understanding of memory impairment related to SVD $[1,13,18,19]$.

Impairment in language functions is not frequent in SVD. Comparing patients with initial dementia, Kertesz et al. found that patients with periventricular hyperintensities performed worse on comprehension and attention tasks compared to those with no hyperintensities, who showed worse performances on memory and conceptualization tasks. However, other studies performed within the general population and with high-risk nondemented patients with WMCs did not find a relationship between the presence of WMCs or lacunes and language deficits. Nevertheless, lexical and semantic fluency measures should be included in the assessment of SVD patients. Impairment in lexical tasks is more frequent and prominent than semantic difficulties and thus showing a different pattern of MCI. As what occurs in other cognitive functional deficits observed in patients with SVD, the type of impairment found in verbal functions seems to be related to the decrease in speed of processing and access to information that affect word-generation tasks. The various neuropsychological batteries utilized were designed to look for impairment in the executive function rather than any other domain $[6,20,21]$.

\section{Radiological Correlates of SVD}

White matter lesions were observed as diffuse areas of white matter attenuation in CT scan and hence termed "leukoaraiosis" derived from the Greek words "leuko-" for "white" and "araios" for "rarefied." SVD-related white matter changes appear as hypodensity in CT and are described as "white matter hypoattenuation" or "white matter hypodensities" but never approach that of cerebrospinal fluid (CSF), as this would indicate complete tissue destruction. Pathologically such areas are characterized primarily by loss of myelin and axons [1]. WMLs tend to be symmetrically distributed throughout the brain with a preference for the periventricular and deep white matter. These lesions are usually 


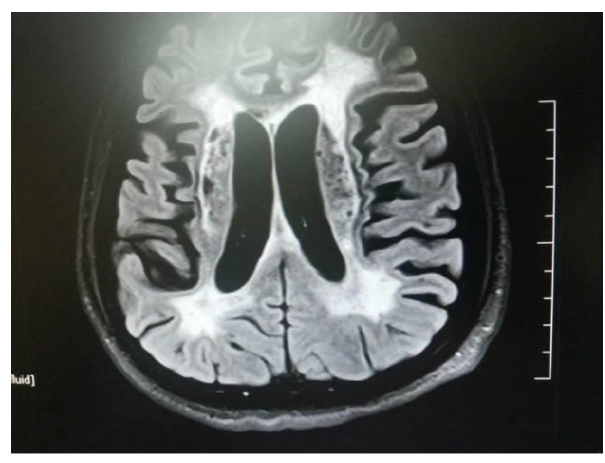

(a) Deep and dense white matter hyperintensities in FLAIR

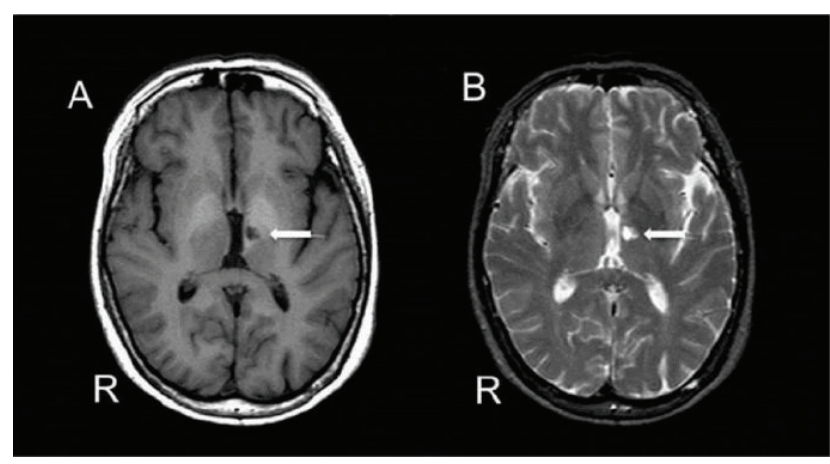

(b) Lacunar stroke. Arrow indicates presence of lacunae in anterior thalamic region

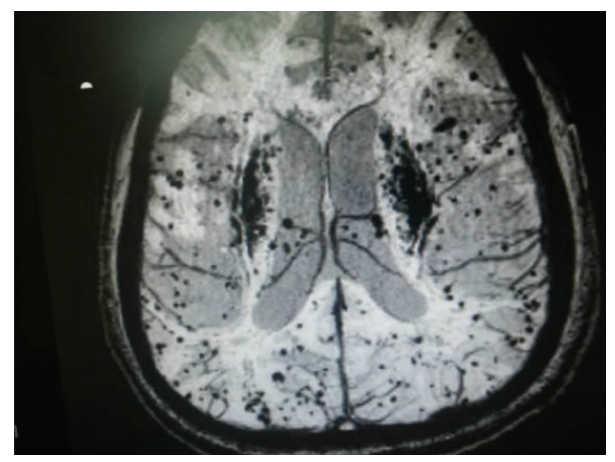

(c) SWI sequence showing diffuse microbleeds

FIGURE 1: (a) FLAIR sequence depicting dense white matter hyperintensities and (b) depicting lacunar strokes; (c) SWI sequence depicting cerebral microbleeds.

ill-defined and characteristically spare the subcortical Ufibers and therefore are easily distinguishable from infarcts which are wedge shaped, are sharply demarcated, extend to the grey matter, may involve the internal capsule, basal ganglia, or thalamus, and follow specific vascular territory and enlargement of the ipsilateral ventricle or sulcus is seen. Plain CT and conventional MR imaging are unable to pick up small acute infarcts in the territory of leukoaraiosis and specific sequences like Diffusion Weighted Images (DWI) often need to be utilized.

MR imaging on the other hand helps to correctly demarcate these lesions and even smaller lesions in thalamus and posterior fossa can be picked up. Fluid attenuation and inversion recovery (FLAIR) sequence is even more helpful and commonly used as it helps in characterizing lacunar infarcts and in distinguishing WMH from enlarged Virchow Robins (perivascular) spaces commonly seen in old age. A form of T2 weighted imaging using gradient echo sequences known as susceptibility weighted imaging is very sensitive in detection of microbleeds and is commonly utilized. SVDrelated WMLs occur preferentially in the deep white matter and close to the lateral ventricles but spare the subcortical arcuate fibers as described above for leukoaraiosis seen on CT.

A number of visual analogue scales to quantify and assess severity of WMH are utilized and fairly good correlation to the WMH is found among the scales $[1,3,5,6,21,22]$ (Table 3 depicts the different visual scales used in SVD).
Many novel methods also include software to compute the volumes of lacunes and $\mathrm{WMH}$ as well as microbleeds which can become a sensitive measure for further research purposes. Magnetization transfer ratio derived susceptibility weighted images are very useful in detection of microbleeds and their characterization [21] (Figures 1(a), 1(b), and 1(c)).

\section{Neuropathological Correlates in SVD}

Fisher was the pioneer in describing the neuropathological details in SVD. From autopsy studies he observed that majority of the lacunes were due to segmental arterial disorganization which implicates patchy, asymmetrical focal vascular changes resulting in the loss of normal architecture and small vessel thickening along with extravasation of plasma proteins into arteriolar wall and subsequent conversion to fibrin known as "lipohyalinosis" and referred to by others as fibrinoid necrosis. These lesions particularly favor the development of spontaneous ICH in deep or infratentorial locations (basal ganglia, thalamus, and pons). The recent work by Pantoni [6] describes the common histopathological lesions in SVD due to hypertensive vasculopathy which includes arteriosclerosis with loss of smooth muscle cells from the tunica media, deposits of fibrohyaline material, luminal stenosis, and thickening of the vessel wall. These features are seen characteristically in aged, hypertensive 
TABLE 3: It depicts different visual scales for CT and MRI which are commonly used.

(a)

\begin{tabular}{|c|c|c|}
\hline $\begin{array}{l}\text { Scales for CT white } \\
\text { matter changes (WMC) }\end{array}$ & Assessment of severity & Scores \\
\hline Van Sweiten et al. & $\begin{array}{l}\text { Severity of WMCs on } 3 \text { subsequent CT slices is graded separately for the regions anterior } \\
\text { and posterior to the central sulcus: } 0=\text { no lesion, } 1=\text { partly involving the white matter, and } 2 \\
=\text { extending up to the subcortical region. The scores for the } 2 \text { regions have to be added } \\
\text { together. }\end{array}$ & $\begin{array}{l}\text { Minimum, 0; } \\
\text { maximum, } 4\end{array}$ \\
\hline Blennow et al. & $\begin{array}{l}\text { Based on extent of WMC } \\
0=\text { no decrease in the attenuation of white matter; } 1=\text { decreased attenuation of white matter } \\
\text { at the margins at the frontal and occipital horns of the lateral ventricles; } 2=\text { decreased } \\
\text { attenuation of white matter around the frontal and occipital horns of the lateral ventricles } \\
\text { with some extension toward the centrum semiovale; and } 3=\text { decreased attenuation of white } \\
\text { matter extending around the whole lateral ventricles and coalescing in the centrum } \\
\text { semiovale. } \\
\text { Based on severity of WMC } \\
0=\text { none, } 1=\text { mild, } 2=\text { moderate, and } 3=\text { marked decrease in the attenuation of white matter. }\end{array}$ & $\begin{array}{l}\text { Minimum, 0; } \\
\text { maximum, } 3 \\
\text { (for extent } \\
\text { and severity } \\
\text { each) }\end{array}$ \\
\hline
\end{tabular}

(b)

Scales for MRI white matter hyperintensities (WMH)

Periventricular and deep WMCs are rated separately. A total score is obtainable by summing the 2 partial scores.

Periventricular hyperintensities

Fazekas et al. Scores are as follows: 0 = absence, 1 = "caps" or pencil-thin lining, $2=$ smooth "halo," and $3=$ irregular periventricular hyperintensities extending into the deep white matter. Deep white matter hyperintense signals Scores are as follows: $0=$ absence, $1=$ punctuate foci, $2=$ beginning confluence of foci, and $3=$ large confluent areas.

Periventricular hyperintensities (minimum, 0; maximum 6) Scoring is as follows: caps, occipital $0 / 1 / 2$, and frontal $0 / 1 / 2$; bands, lateral ventricles $0 / 1 / 2(0=$ absent, $1=\leq 5 \mathrm{~mm}, 2=\geq 6 \mathrm{~mm}$, and $\leq 10 \mathrm{~mm}$ ).

Modified

Scheltens et al. [24]

White matter hyperintensities(minimum, 0 ; maximum, 24)

Scoring is as follows: frontal $0 / 1 / 2 / 3 / 4 / 5 / 6$, parietal $0 / 1 / 2 / 3 / 4 / 5 / 6$, occipital $0 / 1 / 2 / 3 / 4 / 5 / 6$, and

Minimum, 0; temporal $0 / 1 / 2 / 3 / 4 / 5 / 6(0=$ no abnormalities; $1=\leq 3 \mathrm{~mm}, n \leq 5 ; 2=\leq 3 \mathrm{~mm}, n \leq 6 ; 3=4$ to $10 \mathrm{~mm}, n \leq 5 ; 4=4$ to $10 \mathrm{~mm}, n \geq 6 ; 5=\geq 11 \mathrm{~mm}, n \geq 1 ; 6=$ confluent).

WMCs located at 4 locations (frontal horns, body of the ventricles, trigones, and occipital horns) are rated separately on each hemisphere.

Periventricular leukoaraiosis (minimum, 0; maximum, 24)

Scoring is as follows: $0=$ no hyperintensity; $1=$ punctuate, small foci (mild); 2 = cap,

Ylikoski et al. pencil-thin lining (moderate); and 3 = nodular band, extending hyperintensity (severe). Centrum semiovale leukoaraiosis, including watershed areas (minimum, 0; maximum, 24) Scoring is as follows: $0=$ no hyperintensity; $1=$ punctuate, small foci (mild); 2 = beginning confluent (moderate); and $3=$ large confluent areas (severe). Total leukoaraiosis score is periventricular leukoaraiosis score plus centrum semiovale leukoaraiosis score equal to 0 to 48 .

Periventricular and subcortical regions are not rated separately. Including 9 grades: $0=$ no white matter signal abnormalities; 1 = discontinuous periventricular rim or minimal "dots" of subcortical white matter; 2 = thin, continuous periventricular rim or few patches of subcortical white matter lesions; 3 = thicker continuous periventricular rim with scattered patches of subcortical white matter lesions; $4=$ thicker shaggier periventricular rim and mild subcortical white matter lesions and may have minimal confluent periventricular lesions; $5=$ mild, periventricular confluence surrounding frontal and occipital horns; $6=$ moderate periventricular confluence surrounding frontal and occipital horns; $7=$ periventricular confluence with moderate involvement of centrum semiovale; $8=$ periventricular confluence involving most of centrum semiovale; and $9=$ all white matter involved. minimum, 0 ; maximum, 6
Total score

Manolio et al. 
individuals and hence called hypertensive small vessel disease. Other characteristic lesions also include formation of microatheromas in early stage and microaneurysms due to the weakened vessel wall along with poor pliability due to fibrinoid necrosis in later stages. Cerebral white matter damage appears to be also promoted by cerebral amyloid angiopathy (CAA) which preferentially affects the occipital region. CAA is characterized by inflammatory changes with posterior lobar and leptomeningeal vessel involvement with dilated perivascular spaces, cortical siderosis, beta amyloid deposition in the vessel walls, and occasional lobar hemorrhages with coexistence of fresh and old lesions in multiple locations. The word cerebral small vessel disease conventionally stands for the triad of microbleeds, lacunes, and white matter changes. Therefore, the other secondary vascular disorders are not discussed in this review as this is not the primary objective of this paper [24-27].

\section{Treatment}

Treatment options can be grouped into treatment of comorbidities, disease modifying treatment, and symptomatic treatment. Serious comorbidities like substance dependence should be completely stopped. High blood pressure, dyslipidemia, diabetes mellitus, and heart disease should be managed based on a patient oriented way taking into account the severity of the various risk factors, regular monitoring of treatment response, and avoiding certain drugs based on the target organ dysfunction like associated renal and cardiac dysfunction commonly existing in these patients. Elaborate discussion of the pharmacological agents involved is beyond the scope of this paper. Disease modifying treatment with reference to cerebral small vessel disease involves antiplatelet like Aspirin, Clopidogrel, and Dipyridamole based on tolerability and response. Anticoagulants are used when there is evidence for source of embolism like atrial fibrillation, significant heart disease, or atherosclerotic plaques in major vessels like arch of aorta. Neuroprotective agents, homocysteine, and antioxidants are beneficial to some extent. Symptom modifying treatment is chosen based on the symptoms prevalent in the given patient. Patients with cognitive dysfunction are treated with cholinergic drugs and NMDA receptor blockers in addition to cognitive enhancement therapies. If there are seizures, they are treated with anticonvulsants; lower segment parkinsonism which is common in these patients is treated with low dose of Levodopa with decarboxylase inhibitors. Spasticity, aphasia, and dysarthria are treated with antispasticity agents and speech therapy. Occupational rehabilitation is given to patients who have problems in activities of daily living. Dietary advice based on the number and type of comorbidities present is chosen by the dietician based on the occupational demand of the individual $[28,29]$.

\subsection{Single Gene Disorders}

6.1.1. Cerebral Autosomal Dominant Arteriopathy with Subcortical Infarcts and Leukoencephalopathy (CADASIL). It is a hereditary form of SVD causing stroke and WMH with insignificant involvement of the large vessels. It is the most common monogenic condition resulting in SVD. It has nearly $100 \%$ penetrance and is due to mutations of the NOTCH3 gene on chromosome 19 which normally codes for a cell signaling receptor in the vascular smooth muscle cells resulting in uneven production of cysteine residues in the signaling protein leading to abnormal signal transduction and impaired smooth muscle maturation and differentiation. The annual incidence is about $1.5-5 / 100,000$ people and is usually characterized by repeated episodes of migraine with aura, visual field defects, recurrent transient ischemic attacks (TIAs) or strokes, and cognitive impairment usually being detected by the sixth decade. Cognitive impairment is usually manifested as subcortical impairment in attention and retrieval memory and in later stages language and visuospatial disorientation are reported. Epigenetic factors greatly influence the onset of manifestation of illness even in twin studies. Detailed data on these parameters is very scarce and methylation defect is quoted in the study by Brucki et al. [28-30].

MR imaging in CADASIL shows an increased predilection of WMH in the anterior temporal pole, external capsule, and insular region which is not seen in normal ageing. Cerebral atrophy is also seen along with cerebral microbleeds in one third of the patients but deep intracranial hemorrhages are rarely seen. Histopathology is suggestive of a severe small vessel arteriopathy on immunohistological staining characterized by the finding of granular osmophilic inclusions affecting mainly the vascular smooth muscle cells and relative sparing of the endothelium. These pathological findings are remarkably different from normal age related $\mathrm{WMH}$ and deep brain infarcts suggesting an alternative molecular pathophysiology.

Treatment consists of use of oral Aspirin but its efficacy is still not proved in this condition. Cholinesterase inhibitors like donepezil have been tried and proven to have limited role in maintenance of cognition but large trials are still lacking and mainstay of treatment is still supportive measures [28].

6.1.2. Cerebral Autosomal Recessive Arteriopathy with Subcortical Infarcts and Leukoencephalopathy (CARASIL). It is an autosomal recessive disorder with $25 \%$ risk in each pregnancy of heterozygous parents first described in Japanese population. Patients usually present with alopecia beginning in the second decade and spondylosis in the second or third decade. Patient can have early dementia, mood changes, and gait disturbances with spasticity in the lower extremities. It is due to the mutation in the HTRA1 gene which is involved in TGF- $\beta$ signaling. MR imaging findings are similar to that in CADASIL. In addition symmetrical WMH along with signal changes in anterior temporal lobe, cerebellum, brainstem, middle cerebellar peduncle, and external capsule with sparing of the $\mathrm{U}$ fibers is characteristic.

Histopathological picture is suggestive of arteriosclerosis with intimal thickening, loss of smooth muscle cells, and hyaline degeneration resembling characteristic nonhereditary SVD as previously described. Granular osmiophilic material within vascular media adjacent to smooth muscles seen in CADASIL is never seen. 
Treatment is mainly symptomatic and supportive. Use of antiplatelet is recommended along with lifestyle modification and early management of comorbidities especially hypertension $[28,29,31]$.

6.1.3. Hereditary Angiopathy with Nephropathy, Aneurysms, and Muscle Cramps (HANAC). It is an autosomal dominant disease with pathogenic mutation in the COL4A1 gene on chromosome 13 which codes for the alpha-1 subunit of collagen type IV. Affected individuals have microscopic hematuria, muscle cramps with elevated creatine kinase levels, and bilateral retinal artery tortuosity causing repeated retinal hemorrhages. Raynaud's phenomenon and supraventricular arrhythmia are also reported. Diagnosis is made by magnetic resonance brain imaging, featuring frequent leukoaraiosis, subcortical microbleeds, lacunar infarction, and dilated perivascular spaces in conjunction with systemic features or positive family history.

Histopathological assessment reveals irregular abnormal thickening of the basement membrane of the tubules, Bowman's capsule, and interstitial capillaries. Skin biopsy reveals duplication of the basement membrane at the dermoepidermal junction and dermal arterioles. Vascular smooth muscle cells are dissociated due to abnormal spreading of the basement membrane. Treatment is mainly symptomatic but surgical interventions may be required for aneurysms more than $10 \mathrm{~mm}$. COL4A1 gene mutations have been also implicated in autosomal dominant type 1 porencephaly, brain small vessel disease with hemorrhage, and brain small vessel disease with Axenfeld-Rieger anomaly (congenital cataract, microcornea, retinal detachment, increased intraocular pressure, and optic nerve excavation) [26, 27, 32, 33].

6.1.4. Autosomal Dominant Retinal Vasculopathy with Cerebral Leukodystrophy (AD-RVCL). It is an autosomal dominant inherited condition resulting from a frameshift mutation to the TREX1 (three-prime repair exonuclease 1) gene involved in DNA repair on chromosome 3 whose symptoms commonly begin in the early mid-forties. It is associated with progressive loss of vision, migraines, strokes, motor and extrapyramidal features, and cognitive decline. A subset of affected individuals have systemic vascular involvement evidenced by Raynaud's phenomenon, micronodular cirrhosis, and glomerular dysfunction. This disorder is redesignated as CHARIOT (cerebral hereditary angiopathy with vascular retinopathy and impaired organ function caused by TREX1 mutations). Specific treatment options other than antiplatelet are not available [31-34].

6.1.5. Fabry's Disease. It is an X-linked disorder of glycosphingolipid metabolism due to deficient or absent lysosomal alpha galactosidase A activity due to $>300$ types of mutations in the GLA gene (galactosidase alpha). The GLA gene is located in the long arm of X-chromosome at position 22. Glycosphingolipids (mainly globotriaosylceramide, GL3 ) accumulate in lysosomes due to $\alpha$-Gal A deficiency, leading to lysosomal and cell dysfunction; this in turn triggers tissue ischemia and fibrosis. Annual incidence is 1 in 100,000 population. Classically affected patients are males with angiokeratomas, acroparesthesias, hypohidrosis, corneal opacity, and progressive vascular involvement of the brain, heart, and kidneys. There can be recurrent ischemic strokes and painful neuropathies requiring attention. In female carriers high incidence of strokes also has been reported. Microscopic examination of urine will show lipid laden epithelial cells along with serum electrolyte abnormalities. Enzyme analysis will show deficiency of the alpha-galactosidase A enzyme [35].

Treatment includes enzyme replacement therapy (ERT) and Pharmacological Chaperone Therapy (PCT) $[35,36]$.

6.1.6. The Role of Renin-Angiotensin-Aldosterone System. The genes of the renin-angiotensin-aldosterone system (RAAS) system are important candidate genes for SVD as they are the genes controlling hypertension which is the most important risk for SVD. The RAAS is a homeostatic system that affects blood pressure regulation, vasoconstriction, thrombosis, and vessel wall damage. The angiotensin-converting enzyme (ACE), which modulates the proliferation of smooth muscle cells and the tone of vessel walls, may be involved in vascular remodeling and atherosclerosis. It has been proposed that ACE may be a potential determinant for the risk of SVD. An early study reported an association between an ACE insertion-deletion (I/D) polymorphism and lacunar stroke and less or no association with other types of ischemic stroke $[1,17]$. This polymorphism is associated with elevated plasma ACE levels. Similar findings have been shown in some but not all other studies in Caucasian populations [32,33]. A number of studies have also been performed in Asian, predominantly Chinese populations. In this ethnic group, cerebral SVD appears more common [37].

Angiotensinogen is the precursor of angiotensin I. The plasma concentrations of angiotensinogen have been directly related to arterial blood pressure and were found to be modified by variations in the angiotensinogen gene [36]. A missense mutation in exon of the angiotensinogen gene (T704C) encoding threonine instead of methionine at position 235 (M235T) of the amino acid sequence commonly occurs among various ethnic groups. The frequency of the T235 allele ranges from 0.35 in whites to approximately 0.80 in blacks [38]. A large meta-analysis has attributed an increased risk for arterial hypertension to the presence of the T-allele. In comparison with the MM reference group, the excess risk was $31 \%$ in TT homozygotes and $11 \%$ in MT heterozygotes [39]. Studies have found this genetic variant to be associated with coronary artery disease and myocardial infarction [2628]. In a study of SVD, no differences were found in the three promoter $(-18 \mathrm{G}>\mathrm{A},-20 \mathrm{~A}>\mathrm{C}$, and $-6 \mathrm{G}>\mathrm{A})$ and two coding exonic SNPs (174T $>\mathrm{M}$ and $235 \mathrm{M}>\mathrm{T}$ ) or haplotype distribution between cases and control subjects. Amongst hypertensives, however, only the $-20 \mathrm{~A}>\mathrm{C}$ polymorphism was associated with the leukoaraiosis subtype of SVD $[35,36]$. The angiotensin II receptors, which mediate the vasoconstrictive and salt-conserving actions of the RAAS, also represent interesting candidate genes for SVD. Two subtypes of cell 
surface receptors have been identified (AT1 and AT2) using ligand binding studies [40]. The AT1 receptor, through which are exerted most of the actions of angiotensin II, is a G protein-coupled receptor spanning seven transmembrane domains [41, 42]. Several studies suggest that the AT1 receptor might be involved in hypertension. A polymorphism located in the $3^{\prime}$ untranslated region of the ATl receptor gene (AT1) (corresponding to an adenine/cytosine (A/C) base substitution at the 1166 position) has been shown to modify the association of the I/D ACE polymorphism with the occurrence of myocardial infarction [31].

Aldosterone synthase is a key enzyme in the biosynthesis of aldosterone, a mineralocorticoid that regulates arterial function and stimulates arterial fibrosis [41-44]. Studies also indicate that the endogenous aldosterone system may have a role in cerebral SVD $[16,41]$. However, there are no published reports from Indian population on the role of the aldosterone synthase CYP11B2 T-344C polymorphism in vascular cognitive dysfunction associated with SVD $[15,45]$. Thus, gene polymorphisms involved in the RAAS pathway along with aldosterone and ACE levels are of prime interest in regulating the blood pressures and may have a direct influence in the development of cerebral SVD. Knowledge in this area is likely to generate novel treatment options in the management of vascular cognitive impairment.

\section{Conclusions and Future Directions}

Cerebral SVD is a common condition representing about $25 \%$ of all strokes and is of profound importance in view of its probable preventable and treatable nature. The most common risk factor is hypertension which has to be stringently managed. Specific neurochemical pathways which influence blood pressure changes like renin-angiotensinaldosterone system, homocysteine metabolism influencing epigenetic mechanisms in the RAAS genes causing endothelial injury, and the sympathetic autonomic nervous system have a probable role in cognitive decline. A deep insight into these pathways can help in treatment and even prevent this disorder.

\section{Conflict of Interests}

The authors declare that there is no conflict of interests regarding the publication of this paper.

\section{References}

[1] L. Pantoni and P. Gorelick, Cerebral Small Vessel Disease, Cambridge University Press, Cambridge, UK, 1st edition, 2014.

[2] P. Sachdev, Vascular Dementia. The Ageing Brain, 299, 2005.

[3] C. Moran, T. G. Phan, and V. K. Srikanth, "Cerebral small vessel disease: a review of clinical, radiological, and histopathological phenotypes," International Journal of Stroke, vol. 7, no. 1, pp. 3646, 2012.

[4] K. F. De Laat, A. G. W. Van Norden, R. A. R. Gons et al., "Gait in elderly with cerebral small vessel disease," Stroke, vol. 41, no. 8, pp. 1652-1658, 2010.
[5] B. Patel and H. S. Markus, "Magnetic resonance imaging in cerebral small vessel disease and its use as a surrogate disease marker," International Journal of Stroke, vol. 6, no. 1, pp. 47-59, 2011.

[6] L. Pantoni, "Cerebral small vessel disease: from pathogenesis and clinical characteristics to therapeutic challenges," The Lancet Neurology, vol. 9, no. 7, pp. 689-701, 2010.

[7] S. R. Chandra, R. Yadav, C. S. Puneeth, J. Saini, and T. G. Issac, "The spectrum of vascular dementia'-a retrospective study from South India," Journal of Association of Physicians of India, vol. 62, no. 6, pp. 498-503, 2014.

[8] O. Micke, J. Hübner, and K. Münstedt, "Ayurveda," Der Onkologe, vol. 15, no. 8, pp. 792-798, 2009.

[9] L. R. Caplan and J. A. Gomes, "Binswanger disease-an update," Journal of the Neurological Sciences, vol. 299, no. 1-2, pp. 9-10, 2010.

[10] A. P. Palha and M. F. Esteves, "The origin of dementia praecox," Schizophrenia Research, vol. 28, no. 2-3, pp. 99-103, 1997.

[11] A. D. Korczyn, "The complex nosological concept of vascular dementia," Journal of the Neurological Sciences, vol. 203-204, pp. 3-6, 2002.

[12] C. Brayne, C. Gill, F. A. Huppert et al., "Vascular risks and incident dementia: results from a cohort study of the very old," Dementia and Geriatric Cognitive Disorders, vol. 9, no. 3, pp. 175-180, 1998.

[13] G. C. Román, T. Erkinjuntti, A. Wallin, L. Pantoni, and H. C. Chui, "Subcortical ischaemic vascular dementia," The Lancet Neurology, vol. 1, no. 7, pp. 426-436, 2002.

[14] C. Millikan and N. Futrell, "The fallacy of the lacune hypothesis," Stroke, vol. 21, no. 9, pp. 1251-1257, 1990.

[15] J. M. Wardlaw, C. Smith, and M. Dichgans, "Mechanisms of sporadic cerebral small vessel disease: insights from neuroimaging," The Lancet Neurology, vol. 12, no. 5, pp. 483-497, 2013.

[16] D. Brenner, J. Labreuche, F. Pico et al., "The renin-angiotensinaldosterone system in cerebral small vessel disease," Journal of Neurology, vol. 255, no. 7, pp. 993-1000, 2008.

[17] K. Andersen, L. J. Launer, M. E. Dewey et al., "Gender differences in the incidence of $\mathrm{AD}$ and vascular dementia: the EURODEM Studies," Neurology, vol. 53, no. 9, pp. 1992-1997, 1999, AD more in females and no difference in vascular between males and females.

[18] N. D. Prins, E. J. van Dijk, T. den Heijer et al., "Cerebral smallvessel disease and decline in information processing speed, executive function and memory," Brain, vol. 128, no. 9, pp. 20342041, 2005.

[19] G. S. Alexopoulos, B. S. Meyers, R. C. Young, S. Campbell, D. Silbersweig, and M. Charlson, "Vascular depression' hypothesis," Archives of General Psychiatry, vol. 54, no. 10, pp. 915-922, 1997.

[20] W. D. Taylor, H. J. Aizenstein, and G. S. Alexopoulos, “The vascular depression hypothesis: mechanisms linking vascular disease with depression," Molecular Psychiatry, vol. 18, no. 9, pp. 963-974, 2013.

[21] M. Mazzoni, L. Ferroni, L. Lombardi, E. Del Torto, M. Vista, and P. Moretti, "Mini-Mental State Examination (MMSE): sensitivity in an Italian sample of patients with dementia," The Italian Journal of Neurological Sciences, vol. 13, no. 4, pp. 323329, 1992.

[22] T. Erkinjuntti, R. Laaksonen, R. Sulkava, R. Syrjäläinen, and J. Palo, "Neuropsychological differentiation between normal aging, Alzheimer's disease and vascular dementia," Acta Neurologica Scandinavica, vol. 74, no. 5, pp. 393-403, 1986. 
[23] J. T. Moroney, E. Bagiella, D. W. Desmond et al., "Meta-analysis of the Hachinski Ischaemic Score in pathologically verified dementias," Neurology, vol. 49, no. 4, pp. 1096-1105, 1997.

[24] P. Scheltens, T. Erkinjunti, D. Leys et al., "White matter changes on CT and MRI: an overview of visual rating scales," European Neurology, vol. 39, no. 2, pp. 80-89, 1998.

[25] K. A. Jellinger, “The pathology of 'vascular dementia': a critical update," Journal of Alzheimer's Disease, vol. 14, no. 1, pp. 107-123, 2008.

[26] T. Erkinjuntti, G. Román, and S. Gauthier, “Treatment of vascular dementia-evidence from clinical trials with cholinesterase inhibitors," Journal of the Neurological Sciences, vol. 226, no. 1-2, pp. 63-66, 2004.

[27] K. Okamoto, M. Tanaka, and S. Kondo, "Treatment of vascular dementia," Annals of the New York Academy of Sciences, vol. 977, no. 1, pp. 507-512, 2002.

[28] S. M. D. Brucki, A. C. Ferraz, G. R. de Freitas, A. R. Massaro, M. Radanovic, and R. R. Schultz, "Treatment of vascular dementia," Dementia e Neuropsychologia, vol. 5, no. 4, pp. 275-287, 2011.

[29] A. D. Korczyn, V. Vakhapova, and L. T. Grinberg, "Vascular dementia," Journal of the Neurological Sciences, vol. 322, no. 12, pp. 2-10, 2012.

[30] S. Lanfranconi and H. S. Markus, "COL4A1 mutations as a monogenic cause of cerebral small vessel disease: a systematic review," Stroke, vol. 41, no. 8, pp. e513-e518, 2010.

[31] E. Ballabio, A. Bersano, N. Bresolin, and L. Candelise, "Monogenic vessel diseases related to ischemic stroke: a clinical approach," Journal of Cerebral Blood Flow and Metabolism, vol. 27, no. 10, pp. 1649-1662, 2007.

[32] S. Alamowitch, E. Plaisier, P. Favrole et al., "Cerebrovascular disease related to COL4A1 mutations in HANAC syndrome," Neurology, vol. 73, no. 22, pp. 1873-1882, 2009.

[33] P. Ronco and E. Plaisier, "Hereditary angiopathy with nephropathy, aneurysms and muscle cramps (HANAC): a new basement membrane-disease associated with mutations of the COL4A1 gene," Bulletin de l'Academie Nationale de Medecine, vol. 192, pp. 971-986, 2008.

[34] T. Fukutake, "Cerebral autosomal recessive arteriopathy with subcortical infarcts and leukoencephalopathy (CARASIL): from discovery to gene identification," Journal of Stroke and Cerebrovascular Diseases, vol. 20, no. 2, pp. 85-93, 2011.

[35] A. M. Gruver, L. Schoenfield, J. F. Coleman, R. Hajj-Ali, E. R. Rodriguez, and C. D. Tan, "Novel ophthalmic pathology in an autopsy case of autosomal dominant retinal vasculopathy with cerebral leukodystrophy," Journal of Neuro-Ophthalmology, vol. 31, no. 1, pp. 20-24, 2011.

[36] R. P. Grewal and N. W. Barton, "Fabry's disease presenting with stroke," Clinical Neurology and Neurosurgery, vol. 94, no. 2, pp. 177-179, 1992.

[37] M. O. Giwa, J. Williams, K. Elderfield et al., "Neuropathologic evidence of endothelial changes in cerebral small vessel disease," Neurology, vol. 78, no. 3, pp. 167-174, 2012.

[38] X. Jeunemaitre, F. Soubrier, Y. V. Kotelevtsev et al., "Molecular basis of human hypertension: role of angiotensinogen," Cell, vol. 71, no. 1, pp. 169-180, 1992.

[39] S. A. Cooper, A. Whaley-Connell, J. Habibi et al., "Reninangiotensin-aldosterone system and oxidative stress in cardiovascular insulin resistance," American Journal of Physiology: Heart and Circulatory Physiology, vol. 293, no. 4, pp. H2009H2023, 2007.
[40] J. A. Staessen, T. Kuznetsova, J. G. Wang, D. Emelianov, R. Vlietinck, and R. Fagard, "M235T angiotensinogen gene polymorphism and cardiovascular renal risk," Journal of Hypertension, vol. 17, no. 1, pp. 9-17, 1999.

[41] A. T. Chiu, W. F. Herblin, D. E. McCall et al., "Identification of angiotensin II receptor subtypes," Biochemical and Biophysical Research Communications, vol. 165, no. 1, pp. 196-203, 1989.

[42] H. Furuta, D.-F. Guo, and T. Inagami, "Molecular cloning and sequencing of the gene encoding human angiotensin II type I receptor," Biochemical and Biophysical Research Communications, vol. 183, no. 1, pp. 8-13, 1992.

[43] L. Tiret, A. Bonnardeaux, O. Poirier et al., "Synergistic effects of angiotensin-converting enzyme and angiotensin-II type 1 receptor gene polymorphisms on risk of myocardial infarction," Lancet, vol. 344, no. 8927, pp. 910-913, 1994.

[44] J. M. C. Connell, R. Fraser, S. MacKenzie, and E. Davies, "Is altered adrenal steroid biosynthesis a key intermediate phenotype in hypertension?" Hypertension, vol. 41, no. 5, pp. 993-999, 2003.

[45] M. J. E. Van Rijn, M. J. Bos, A. Isaacs et al., "Polymorphisms of the renin-angiotensin system are associated with blood pressure, atherosclerosis and cerebral white matter pathology," Journal of Neurology, Neurosurgery \& Psychiatry, vol. 78, no. 10, pp. 1083-1087, 2007. 


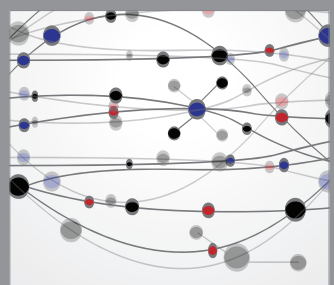

The Scientific World Journal
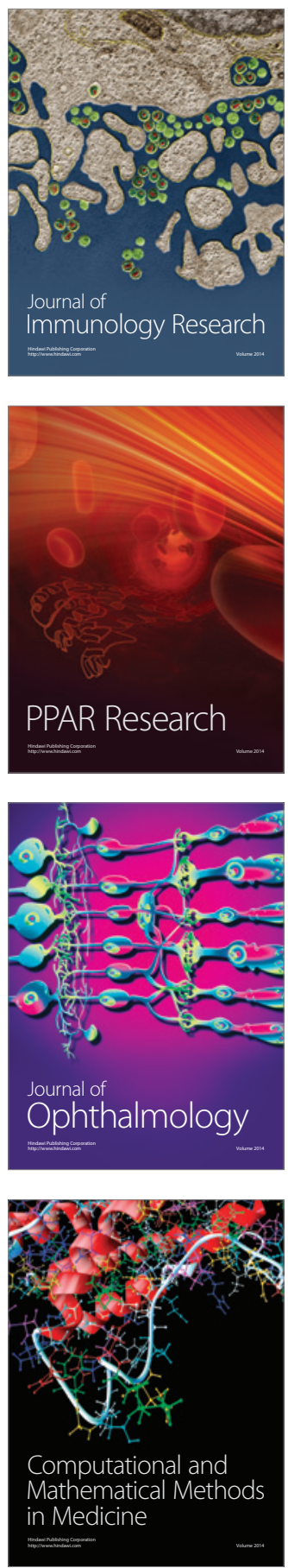

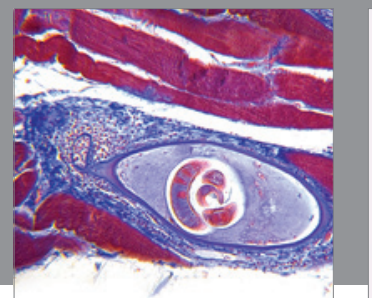

Gastroenterology

Research and Practice
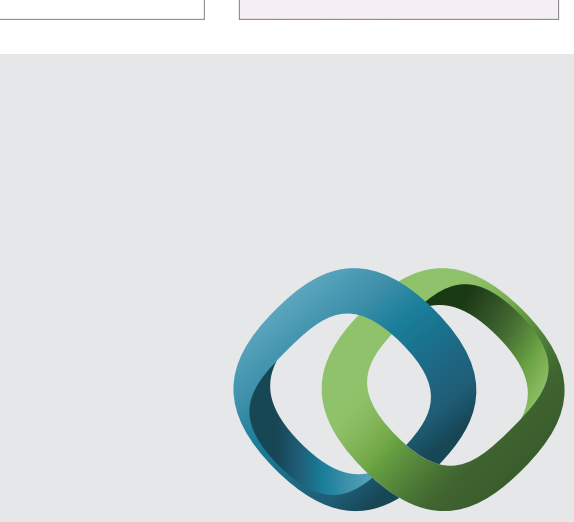

\section{Hindawi}

Submit your manuscripts at

http://www.hindawi.com
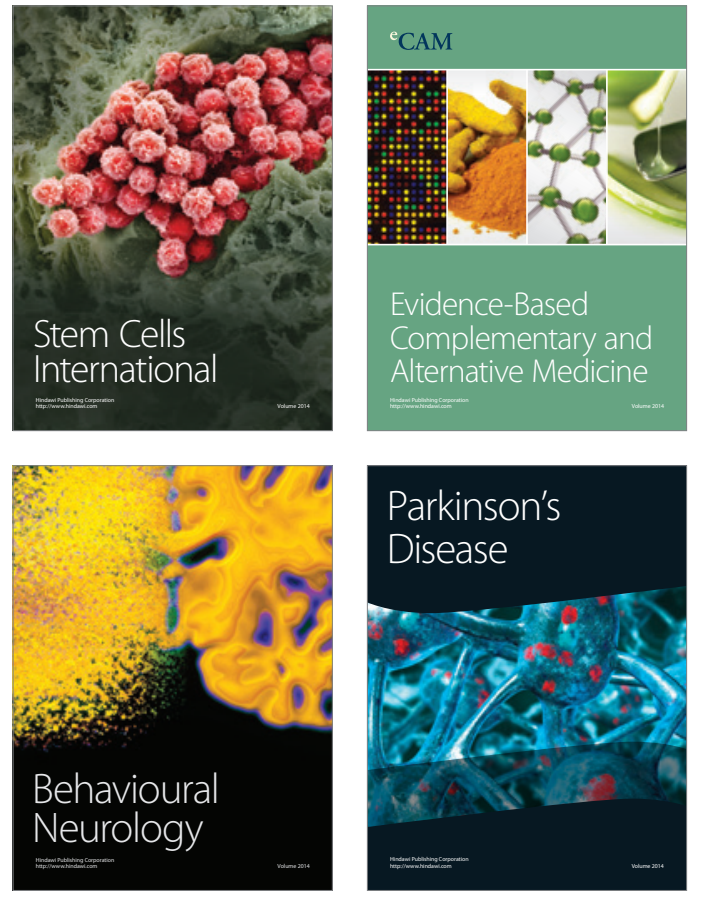
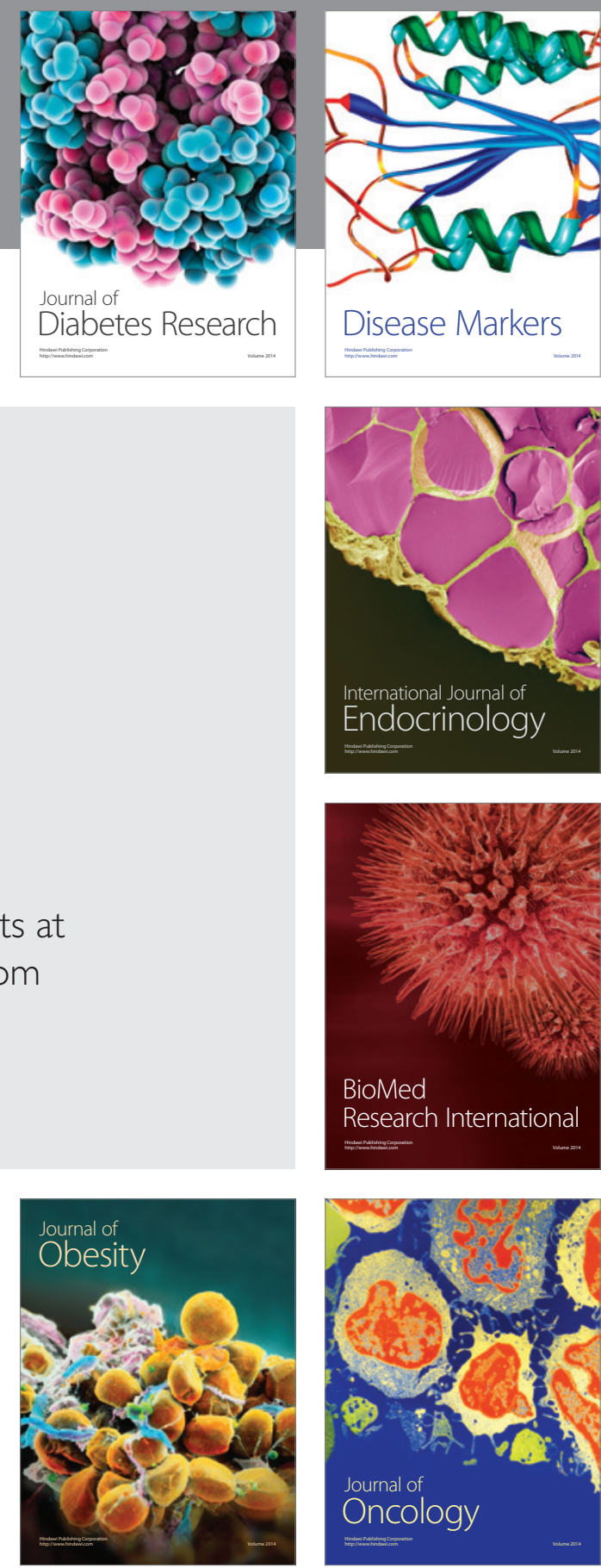

Disease Markers
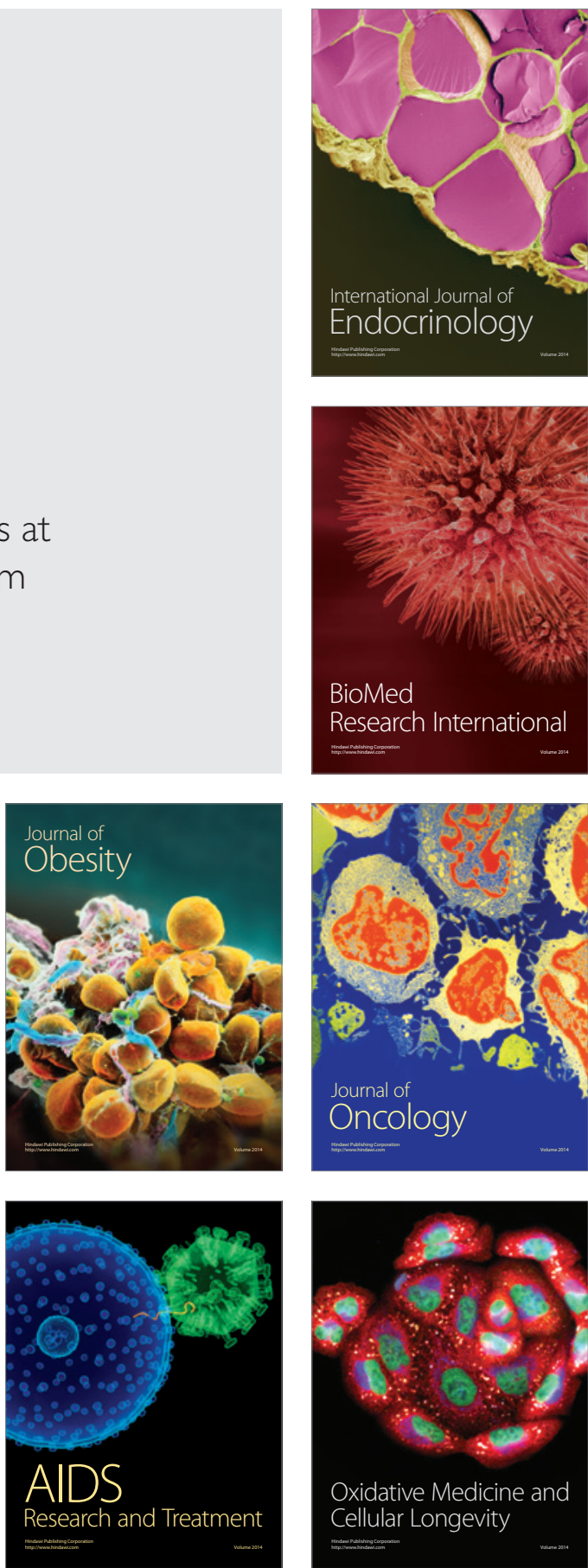